Vol.1 No.1, Spring 2020

\title{
Awareness about warning signs for cancers in women referred to health centers affiliated with bushehr university of medical sciences
}

\author{
Sharifikia $\mathrm{I}^{1}$,Rohani $\mathrm{C}^{2 *}$, Estebsari $\mathrm{F}^{3}$, Salmani $\mathrm{F}^{4}$, Matbouei $\mathrm{M}^{5}$, Hossein-nejad $\mathrm{A}^{6}$, Afrasiabi $\mathrm{S}^{7}$
}

\begin{abstract}
(1)
CrossMark

dick for updates

1- MSc in Community Health Nursing, School of Nursing and Midwifery, Shahid Beheshti University of Medical Sciences, Tehran, Iran 2- Assistant Professor, Department of Health, School of Nursing and Midwifery, Shahid Beheshti University of Medical Sciences, Tehran, Iran 3-Assistant Professor, Department of Health, School of Nursing and Midwifery, Shahid Beheshti University of Medical Sciences, Tehran, Iran 4-PhD. in Biostatistics, Department of Biostatistics, School of Allied Medical Sciences, Shahid Beheshti University of Medical Sciences, Tehran, Iran

5-MSc. in Medical-Surgical Nursing, Department of Health, School of Nursing \& Midwifery, Shahid Beheshti University of Medical Sciences, Tehran, Iran

6-MSc. in Community Health Nursing, Department of Nursing, School of Nursing and Midwifery, Bushehr University of Medical Sciences, Bushehr, Iran

7- MSc. in Midwiferry, Department of Midwifery, School of Nursing and Midwifery, Bushehr University of Medical Sciences, Bushehr, Iran Corresponding Author: Rohani C, Assistant Professor, Department of Health, School of Nursing and Midwifery, Shahid Beheshti University of Medical Sciences, Tehran, Iran
\end{abstract}

E-mail:camelia.rohani@sbmu.ac.ir

Received: 5 April 2019

Accepted: 29 May 2019

\section{Abstract}

Introduction: The number of new cancer cases in the world and in developing countries such as Iran is still growing. Success in early detection of cancer largely depends on people's awareness of the early warning signs of cancers. This study aimed to determine the level of women's awareness about the warning signs of cancers and its association with demographic and clinical characteristics.

Method: This descriptive cross-sectional study was carried out on 160 women referred to the four health centers affiliated with the Bushehr University of Medical Sciences. The data were obtained using two researchers-made questionnaires, including demographic-clinical characteristics questionnaire and questionnaire of awareness for cancer signs.

Results: The mean age of women in this study was $29.9 \pm 7.1$ years. According to the results, over $50 \%$ of women were not aware of five signs out of 10 warning signs of cancer. More than half of the women (56.2\%) had a low level of awareness and poor knowledge of cancer warning signs. The results of linear regression analysis showed that only the women's level of education was significantly associated with their awareness of the warning signs of cancer ( $p=0.007, \beta=0.28$ ). In total, $11 \%$ of the variations in women's awareness of the warning signs of cancer were predicted by this variable.

Conclusion: considering the low level of knowledge of women about the warning signs of cancers, there is an important need for community/ public health nurses in the community. In addition, the findings reinforce need for implementing intervention programs from school phase to adulthood supported by health policy makers.

\begin{tabular}{|l|l|}
\hline \multicolumn{3}{|c|}{ Access this article online } \\
\hline
\end{tabular}




\section{بررسى ميزان آكاهى زنان مراجعه كننده به مر اكز بهداشتى -درمانى تحت يوشش دانشكاه علوم يزشكى بوشهر درباره علايم هشدار دهنده سرطان هانهان}



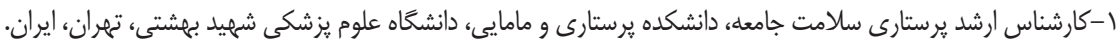

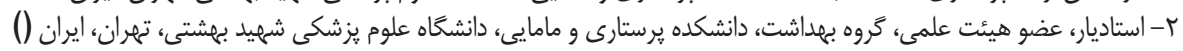

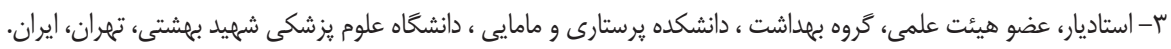

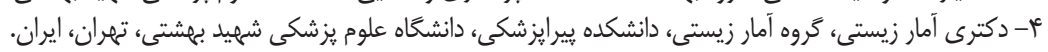

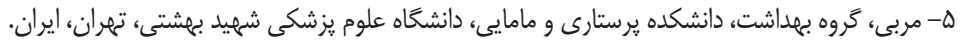

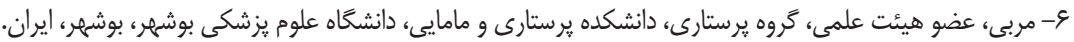

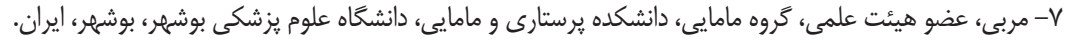

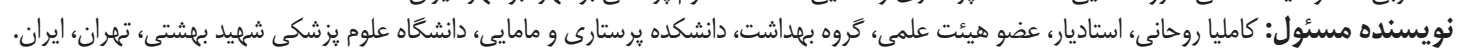
ايميل:Samelia.rohani@sbmu.ac.ir

مقدمه: هنوز تعداد موارد سرطان در جهان رو به افزايش بوده و در كشورهاى در حال رشد از جمله ايران با روندى رو به رشد مواجه است. موفقيت در

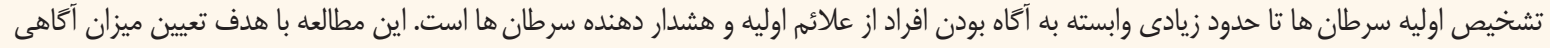

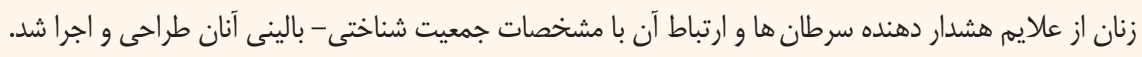

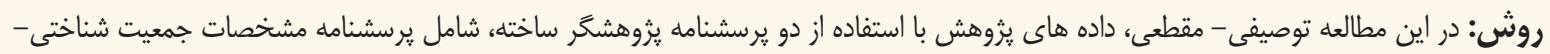

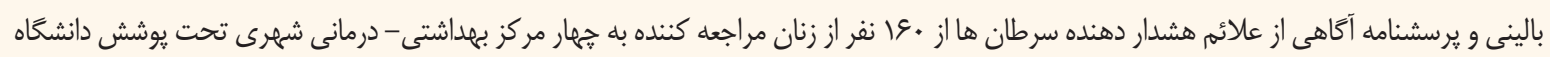
علوم يزشكى بوشهر جمع آورى شد.

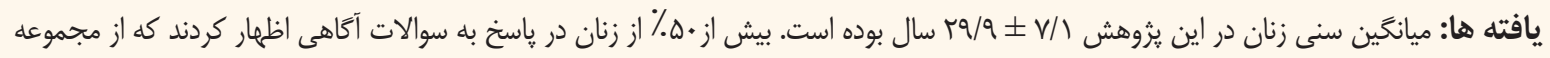

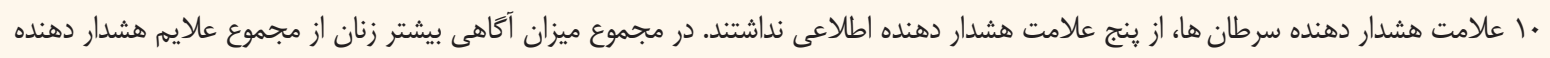

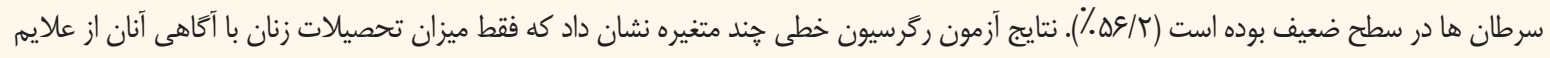

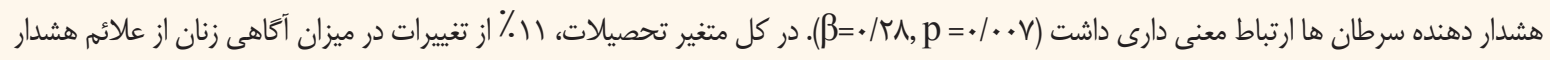
دهنده سرطان ها را ييش بينى كرد.

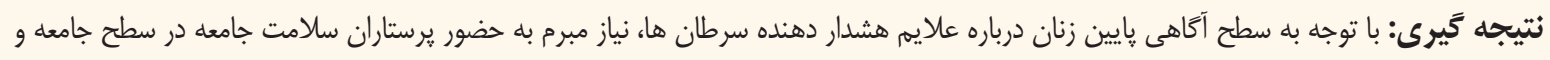

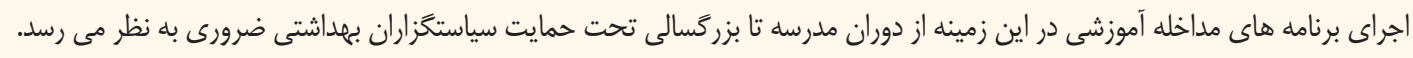




تغييرات قابل ملاحظه در خال ها و زَيل ها، سرفه هاى مكرر و يا



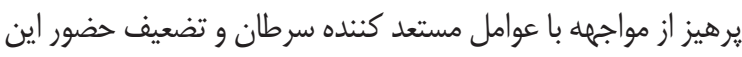

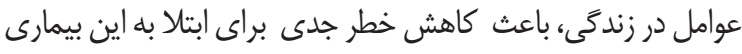

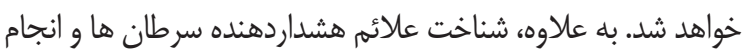







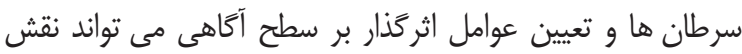

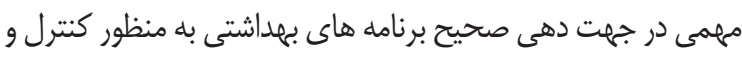

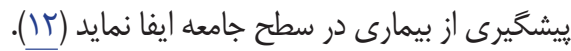





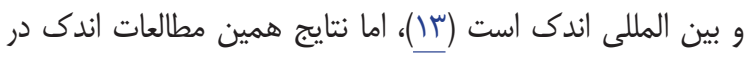



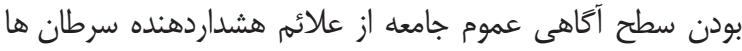

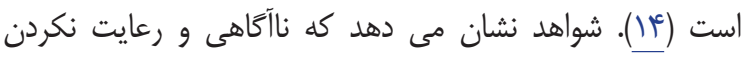
رفتارهاى صحيح بهداشتى در هر جامعل اجتناب نايذير است، و افر إداد و جوامع براى شناخت شيوه هاى صحيح زندى ونى و كاربرد آن و يرهيز

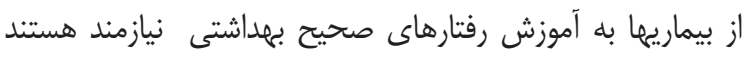

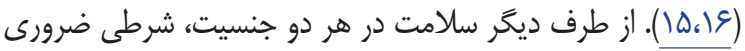



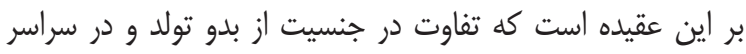



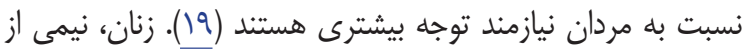

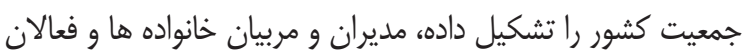



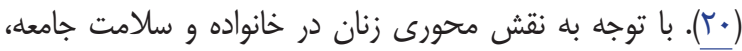

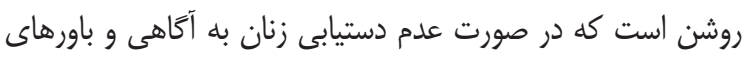



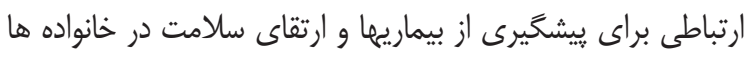

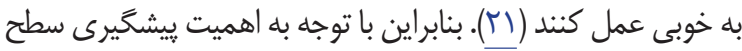

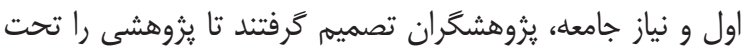

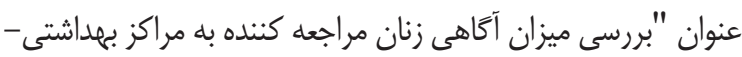

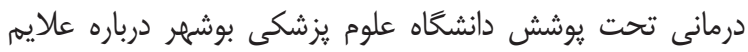
هشدار دهنده سرطان ها" انجام دهند.

\section{روش مطالعه}

اين يزوهش يك مطالعه اوليه با طرح توصيفى - مقطعى است

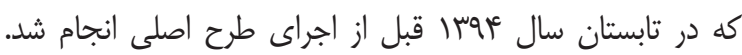

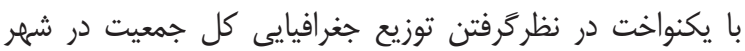



\section{مقلدمه}

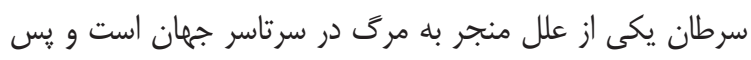

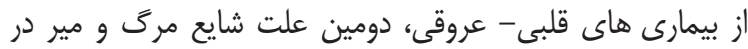

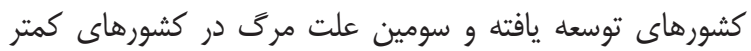

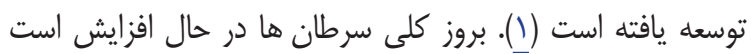

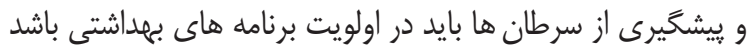

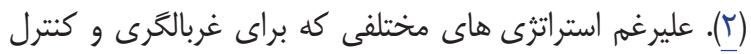

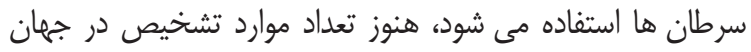

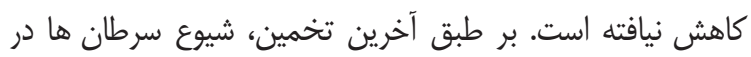

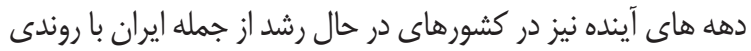

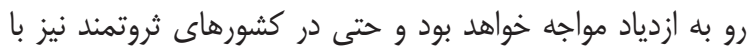

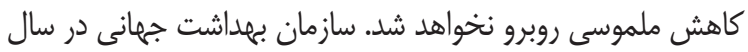

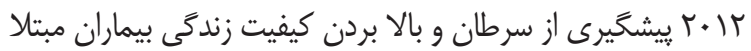

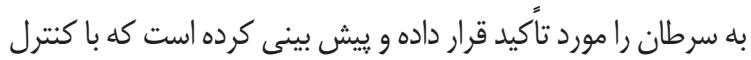

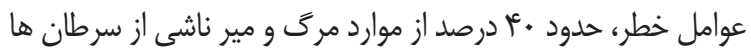

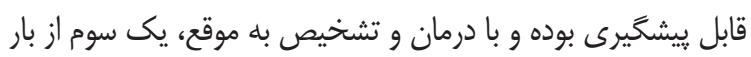

بيمارى كاهش مى يابد (با).

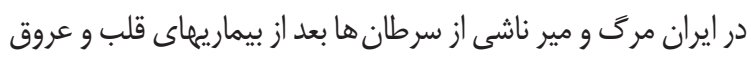

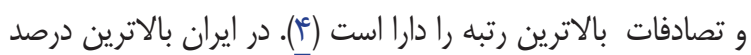

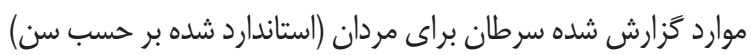

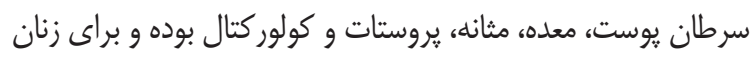

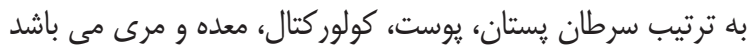

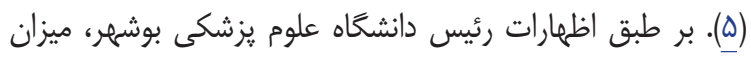

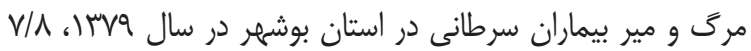

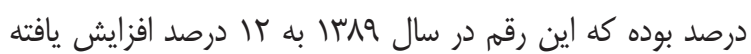
است. بدين ترتيب سرطان عامل اول و اصلى مركى و و مير در استان

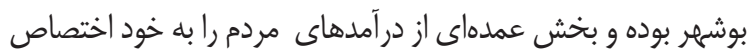

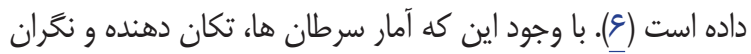

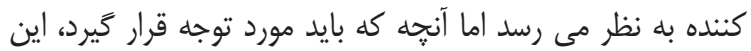

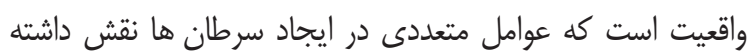

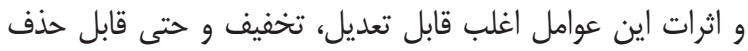



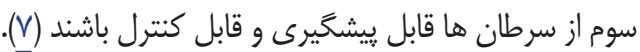

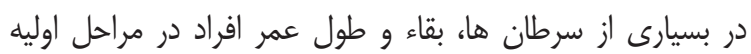

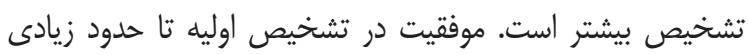

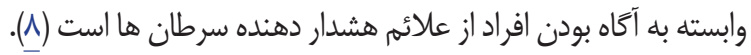

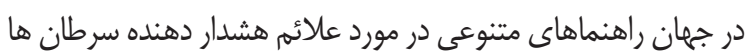



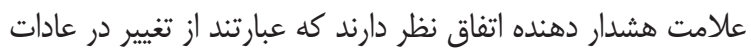

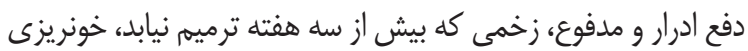

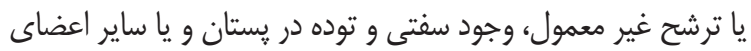
بدن، اشكال در بلع، اشكال در هضم و جذب غذان تذا (سوء هاضمه)، 
يرسشنامه يزوهشكَر ساخته آَاهى مبتنى بر نظرات متخصصين

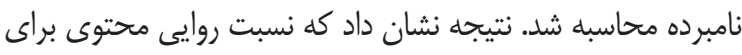

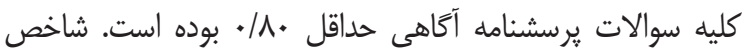

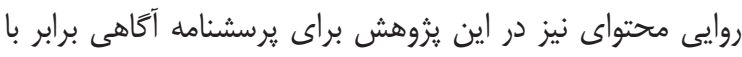

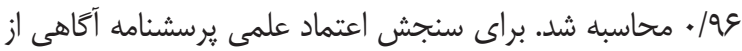

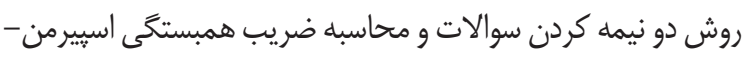

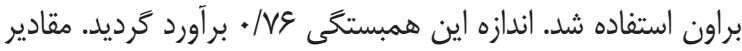

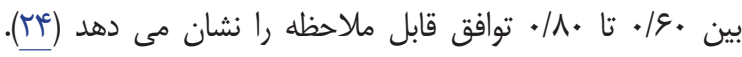

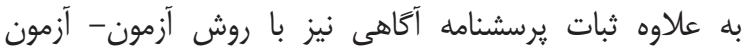

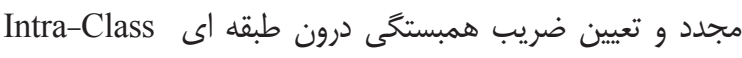
Correlation Coefficient: ICC

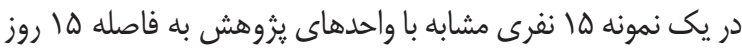

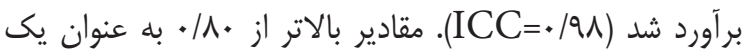

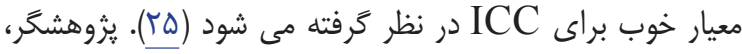

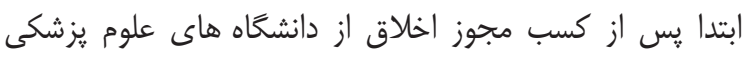
شهيد بهشتى و بوشهر و مراكز بهداشتى - درمانى تابعه دانشكاه

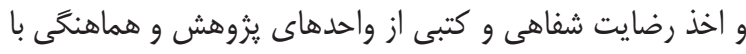

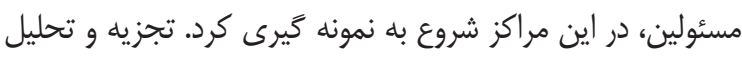



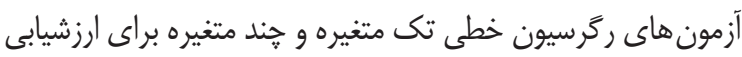
نحوه اثر كذارى متغيرهاى مستقل جمعيت شناختى - بالينى بر ميزان

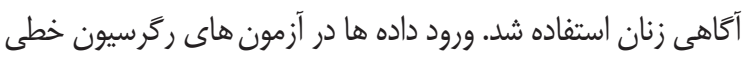

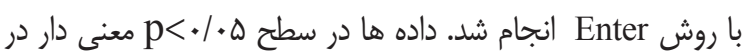



\section{يافته ها}



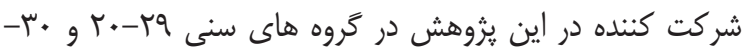





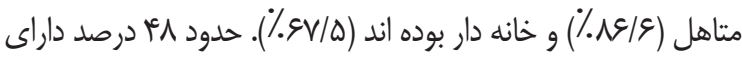
تحصيلات دانشخاهى بوده و به اظهار خودشان اكثر آنها درباره

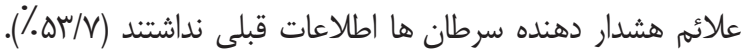
بيشترين منبع كسب اطلاعات زنان از علائم هشداردهنده سرطان ها، رسانه ها (كتاب، روزنامه و مجالات، ايتترنت، راديو، تلويزيون، ....) بوده است (ץ// و٪). ساير اطلاعات جمعيت شناختى و بالينى در جداول او ك نشان داده شده است.
شهرستان بوشهر و تحت يوشش دانشكاه علوم يزشكى بوشهر (11)

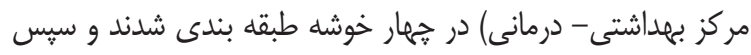

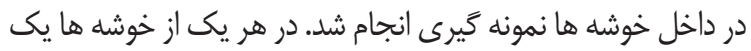
مركز بهداشتى - درمانى به طور تصادفى انتخاب شد. در داخل مراكز

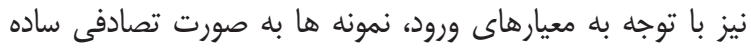

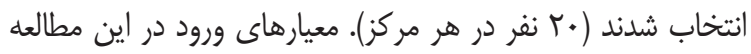
عبارت بودند از زنانى كه به مراكز بهداشتى - درمانى شهرى تحت

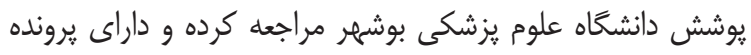
بهداشتى خانوار باشند. اين زنان علاوه بر سواد خواندن و نوشتن، بايد بايد

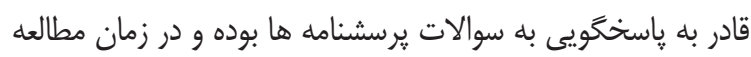

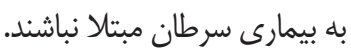
در اين مطالعه اوليه، جهت تعيين حجم نمونه با استفاده از جداول Cohen

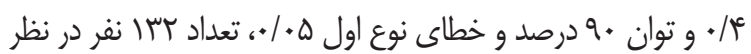

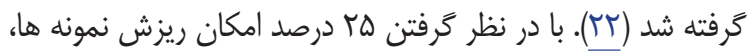

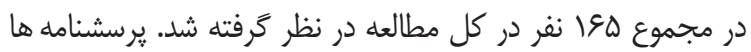

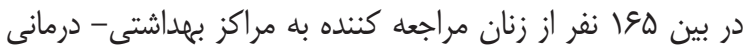

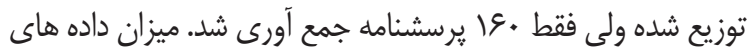

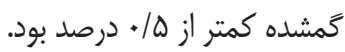
داده هاى مطالعه با استفاده از دو برسشنامه يزوهششَّر ساخته شامل يرسشنامه اطلاعات جمعيت شناختى- بالينى با وأ سوال (سن،

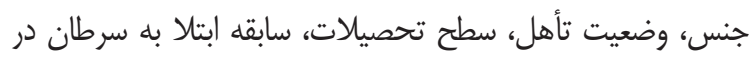
اعضاى نزديك خانواده، مصرف دخانيات و مصرف الكل و شناخت علائم هشدار دهنده سرطان) و يرسشنامه آكاهى از علائم هشدار

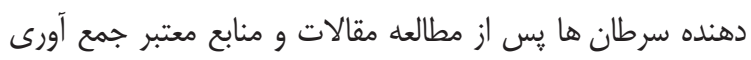

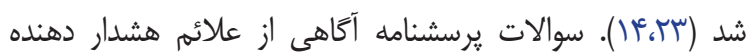



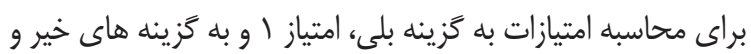



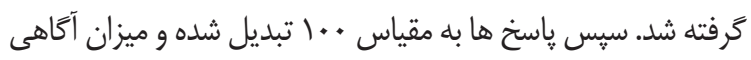
زنان از علايم هشدار دهنده سرطان ها با توجه به ميانكين و نظر

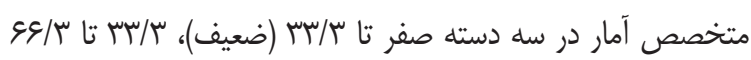

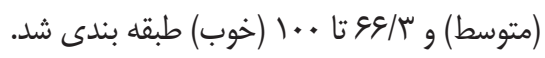
جهت تعيين روايى يرسشنامه ها از روايى صورى و روايى محتوى كيفى توسط • ا نفر از متخصصين و اعضاى هيات علمى دانشكده

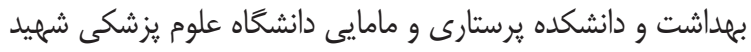

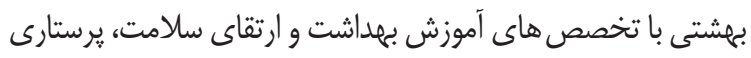
بهاشت جامعل، روانيرستارى و يرستارى داخلى - جراحى استفاده شد. همجنين نسبت روايى محتوى (Content Validity Ratio: CVR) و شاخص روايى محتوى (Content Validity Index: CVI) براى 
جدول (: مشخصات جمعيت شناختى زنان شركت كننده در يثوهش (•l(n=)

\begin{tabular}{|c|c|c|c|}
\hline درصد & تعداد & \multicolumn{2}{|c|}{ متغيرهاى جمعيت شناختى } \\
\hline $\begin{array}{l}V / q \\
1 \varepsilon / 9 \\
r / r \\
1 / r\end{array}$ & $\begin{array}{c}19 \\
179 \\
9 \\
19\end{array}$ & همسر هناهل هدرد & 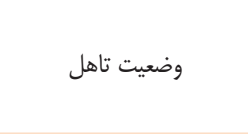 \\
\hline  & $\begin{array}{c}v \\
r I \\
1 \\
\text { rq } \\
v V\end{array}$ & 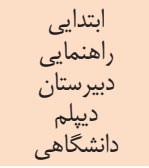 & ميزان تحصيلات \\
\hline $\begin{array}{l}m / / q \\
g V / \Delta \\
\cdot / \varphi\end{array}$ & $\begin{array}{c}01 \\
1+1 \\
1\end{array}$ & خازنشه دار & وضعيت اشتغال \\
\hline $\begin{array}{l}g / r \\
11 / \Lambda \\
g / r \\
r \Delta / 9 \\
0 \cdot 1 .\end{array}$ & $\begin{array}{l}1 . \\
19 \\
1 . \\
11 \\
1 .\end{array}$ & داهنمايتى دابيى دابيلمان & ميزان تحصيلات همسر \\
\hline $\begin{array}{l}9 \cdot 1 \cdot \\
1 / 9 \\
1 / 1\end{array}$ &  & بازنشسته & وضعيت اشتغال همسر \\
\hline $\begin{array}{l}r T / \Delta \\
S V / \Delta\end{array}$ & $\begin{array}{l}\Delta T \\
1.1\end{array}$ & خبي & كفايت در آمد ماهيانه خانواده \\
\hline
\end{tabular}

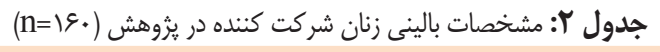

\begin{tabular}{|c|c|c|c|}
\hline درصد & ت تعداد & & متغير بالينح \\
\hline $\begin{array}{l}r \cdot / q \\
r q / 4\end{array}$ & 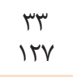 & 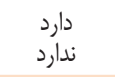 & سابقه خانوادگى ابتلا به سرطان \\
\hline $\begin{array}{l}f I / r \\
\Delta N / V\end{array}$ & $\begin{array}{l}V^{c} \\
\wedge \varepsilon\end{array}$ & خيلى & كسب اطلاعات قبلى از علائم \\
\hline $\begin{array}{l}m I / r \\
\Delta q / \mu \\
q /{ }^{c}\end{array}$ & $\begin{array}{l}\Delta . \\
90 \\
10\end{array}$ & تاحدودى بلى & رعايت تغذيه صحيح \\
\hline $\begin{array}{l}N / 1 \\
V / \Delta \\
\Lambda F / /^{c}\end{array}$ & 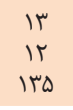 & خاحدودى خلى & مصرف دخانيات \\
\hline $\begin{array}{l}\text { M } \\
r / \Lambda / \Lambda \\
r / \Lambda\end{array}$ & $\begin{array}{l}\Delta Y \\
\Delta F \\
\Delta F\end{array}$ & تاحدودى بلى & انجام ورزش روزانه \\
\hline $\begin{array}{l}N / 1 \\
V / \Delta \\
\Lambda F / T^{\leftarrow}\end{array}$ & $\begin{array}{l}\text { it } \\
\text { it }\end{array}$ & خليدى خلى خلى & مصرف دخانيات \\
\hline $\begin{array}{l}q / 9 \\
k / q^{c} \\
M / V\end{array}$ & $\begin{array}{l}11 \\
8 \\
1145\end{array}$ & خاحدودى خيى & مصرف الكل \\
\hline $\begin{array}{l}. / 8 \\
1 / \Lambda \\
r . / . \\
r / / \phi \\
1 N / 1\end{array}$ & $\begin{array}{l}1 \\
\text { If } \\
\text { gr } \\
\Delta r \\
\text { rq }\end{array}$ & ختوسط & ارزيابى وضعيت سلامت \\
\hline $\begin{array}{l}\Delta / \cdot \\
r \cdot / \cdot \\
\varphi \Delta / \cdot\end{array}$ & $\begin{array}{l}v \\
4 q \\
1 \cdot p\end{array}$ & زمتوسط & ميزان توجه به سلامتى \\
\hline $\begin{array}{l}V / \Delta \\
q Y / Q\end{array}$ & If & 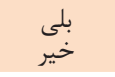 & ارزيابى وضعيت سلامت \\
\hline
\end{tabular}




\section{ايمان شريفى كيا و همكاران}

در عادات دفع ادرار و مدفوع (ه1\%) و اشكال در هضم و جذب غذا (سوء هاضمه) (ها \%) در مقايسه با بقيه علايم هشدار دهنده سرطان

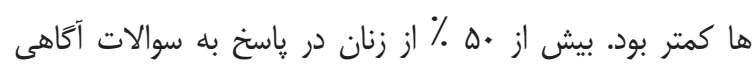

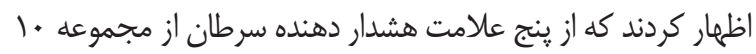
علامت هشدار دهنده، اطلاعى نداشتند (گزينه نمى دانم).
در جدول ب كليه سوالات زيرسشنامه آكَاهى از علايم هشدار دهنده

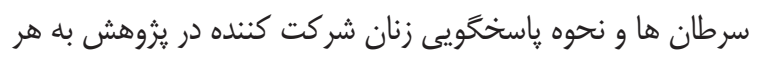

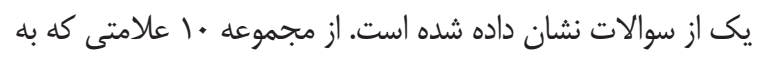
عنوان علائم هشداردهنده سرطان ها مطرح شده اند، بيشتر زنان بيان كردند كه از علامت سفتى و وجود توده در يستان و يا ساير اعضاى

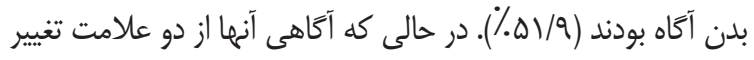

جدول با: فراوانى ياسخكَويى زنان به سوالات يرسشنامه آَاهى از عالائم هشار دهنده سرطان ها

\begin{tabular}{|c|c|c|c|}
\hline درصد & 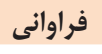 & 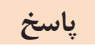 & علائم هشدار دهنده سرطان ها \\
\hline $\begin{array}{l}r / \Lambda \\
\Delta r / 1 \\
\mid r / 1\end{array}$ & $\begin{array}{l}\Delta f \\
\Lambda \Delta \\
r\end{array}$ & نمى ديلى & تغييرات قابل زملاحظه در خال ها و \\
\hline $\begin{array}{l}r \Delta / \cdot \\
I V / \Delta \\
\tau / / \Delta\end{array}$ & $\begin{array}{l}\Delta G \\
\text { YA } \\
\text { Vq }\end{array}$ & نمى دير منم & كاهش وزن نامعانى \\
\hline $\begin{array}{l}r / / \mu \\
r \mu / V \\
\Delta \Delta / \cdot\end{array}$ & $\begin{array}{l}r F \\
\mu \Lambda \\
M\end{array}$ & 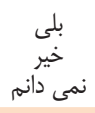 & زخمى كه بيش از سه هفته بهبود و \\
\hline $\begin{array}{l}r q / \Lambda \\
r / / \mu \\
r q / 9\end{array}$ & $\begin{array}{l}\text { qT } \\
\text { qu } \\
\text { VQ }\end{array}$ & نمى ديلى & خونريزى يا ترشح غير معمول \\
\hline $\begin{array}{l}1 Q / / \\
T V / \Delta \\
\Delta V / \Delta\end{array}$ & $\begin{array}{l}\text { re } \\
4 q \\
q \mu\end{array}$ &  & تغيير در عادات دفع ادرار و مدفوع \\
\hline $\begin{array}{l}r T / \Delta \\
T / \Delta / \Delta \\
T \Delta / .\end{array}$ & $\begin{array}{l}\Delta r \\
r q \\
v r\end{array}$ & نمى دير دئم & اشكال در بلع غذا \\
\hline $\begin{array}{l}01 / 9 \\
19 / 4 \\
Y N / V\end{array}$ & $\begin{array}{l}\mu r \\
\mu \\
r q\end{array}$ & نمى ديلى & سفتى و وجود توده ايى در پِتان و يا \\
\hline $\begin{array}{l}10 / \% \\
r q / \bar{c} \\
\Delta \omega / \bar{q}\end{array}$ & $\begin{array}{l}r F \\
k V \\
19\end{array}$ & نمى دير & شكال در هضم و جذب غذا (سوء هاضمه) \\
\hline $\begin{array}{l}r . / . \\
r g / q \\
\Delta r / 1\end{array}$ & $\begin{array}{l}\text { Tr } \\
\text { ra } \\
\text { Ta }\end{array}$ & نمى دير دانم & 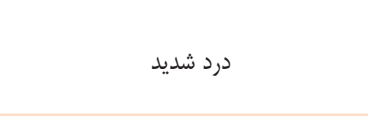 \\
\hline $\begin{array}{l}18 / 9 \\
r \cdot / 9 \\
\Delta r / \Delta\end{array}$ & $\begin{array}{l}\text { hi } \\
\text { kq } \\
\text { iq }\end{array}$ & نمى دير & سرفه هاى مكرر و ويا خشونت و تغيير \\
\hline
\end{tabular}

طبقه بندى ميزان آكَاهى زنان در سه دسته ضعيف و متوسط و خوب ميزان آكَاهى بيشتر زنان از علايمم هشدار دهنده سرطان ها در سطح

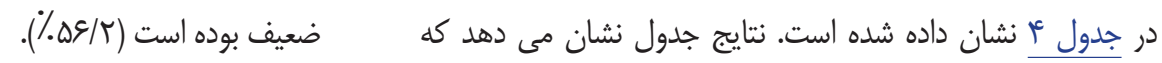
جدول أ: طبقه بندى امتيازات ميزان آكاهى زنان از علايمم هشدار دهنده سرطان ها

\begin{tabular}{|c|c|c|}
\hline درصد & فراوانى & آَاهى \\
\hline$\Delta \varepsilon / T$ & q. & ضعيف \\
\hline س/ץ & $\Delta \Lambda$ & متوسط \\
\hline$V / \Delta$ & Ir & خوب \\
\hline $1 \ldots$ & $\begin{array}{c}19 . \\
r V / .9 \pm|N / D|\end{array}$ & ميانكين و انحراف معيار \\
\hline
\end{tabular}

متغيره بين اين متغيرها، متغيرهاى مستقل معنى دار انتخاب شدند.

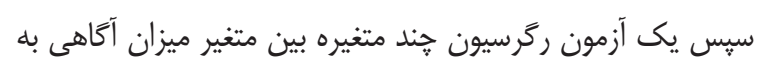

براى بررسى رابطه ميزان آكَاهى و متغيرهاى جمعيت شناختى

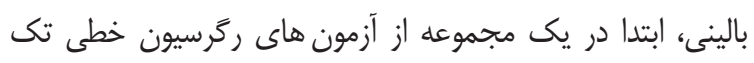


معنى كه با بالا رفتن سطح تحصيلات زنان، ميزان آكاهى آنان نيز از علايم هشدار دهنده سرطان ها افزايش يافت. اين متغير در

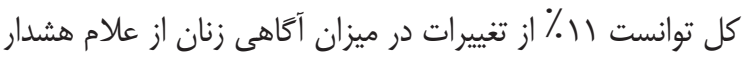

دهنده سرطان ها را ييش بينى كند.
عنوان متغير وابسته و متغير هاى معنى دار جمعيت شناختى - بالينى

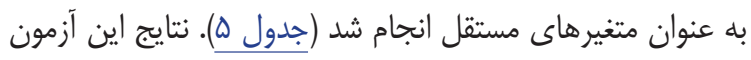
نشان داد كه پِ از كنترل متغيرهاى جمعيت شناختى- بالينى،

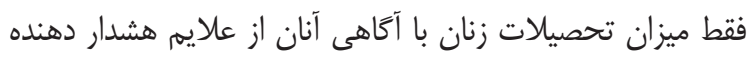

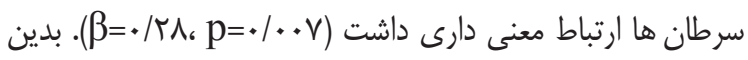
جدول ه: نتايج مدل ركَرسيون خطى جندكانه بين متغير آكاهى زنان از علايم هشدار دهنده سرطان ها و متغيرهاى جمعيت شناختى - بالينى

متغير وابسته : آكاهى

\begin{tabular}{|c|c|c|c|c|}
\hline $\mathbf{P}$ & $\mathbf{t}$ & استاندارد & | استاندارد & متغير مستقل شاخص آمارى \\
\hline ./rq. & I/ITE & $r / M r$. & .1 .94 & وضعيت تاهل \\
\hline.$/ . v$ & T/VTV & $r / \cdots V$ & . TNE & سطح تحصيلات \\
\hline 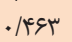 &.$- / V R \Delta$ & $1 / .14$ & -.1 .90 & وضعيت اشتغال \\
\hline . $/ 4 T$ & $-1 / T \Delta V$ &.$/ 095$ &.$- / 14$ & سطح تحصيلات همسر \\
\hline . /AMF & - - &.$/ \Delta V T$ &.$- / \cdot 1 f$ & وضعيت اشتغال همسر \\
\hline ./rq४ & - &.$/ A V t$ & $-\cdot / \cdot \wedge \Delta$ & سابقه خانوادگى ابتلا به سرطان \\
\hline.$/ 119$ & $-r /$ rag &.$/ T V \Delta$ &.$- / 194$ & كسب اطلاعات قلد \\
\hline 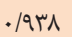 &.$/ \cdot r$ &.$/ v \wedge 1$ & $.1 . .9$ & ارزيابي وضعيت سلامت \\
\hline - IV & $-1 / r 99$ &.$/ 891$ & 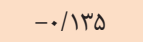 & ميزان توجه به سلامت \\
\hline \multicolumn{4}{|c|}{.11 .9} & R Square \\
\hline
\end{tabular}

مردم درباره بعضى از ريسك فاكتورها مثل سيكار و الكل بالاتر از

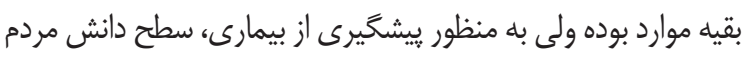

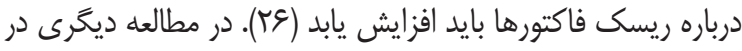

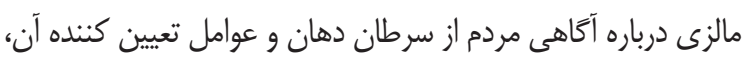

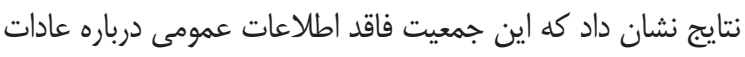
مخاطره آميز، نشانه ها و علايم اوليه و فوايد كشف بيمارى در مراحل

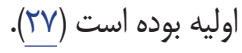

نتايج مدل ركرسيون خطى خُند متغيره در اين مطالعه نشان داد

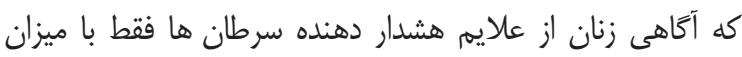

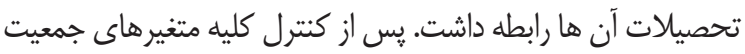
شناختى -بالينى، سطح تحصيلات زنان حدود /ل الدرصد از تغييرات

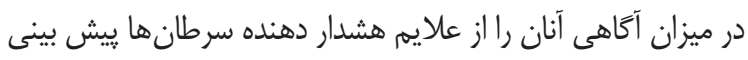

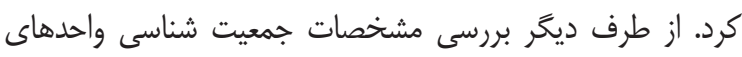
يزووهش نشان داد كه حدود مثأ درصد از زنان مر اجعه كننده به مراكز بهداشتى - درمانى تحصيلات دانشخاهى داشته اند. مطالعات مختلف

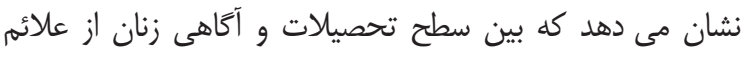
هشدار دهنده سرطان ها ارتباط وجود دارد. بدين ترتيب كه با افزايش لـان سطح تحصيلات، ميزان آكاهى واحدهاى يزوهش نسبت بـاط به عالائم

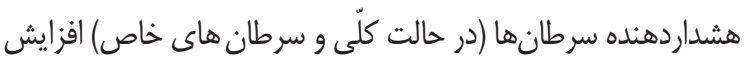

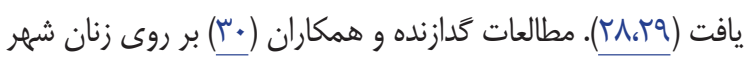

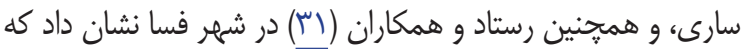
به ترتيب سطح آكَاهى زنان در مورد سرطان بِتان و برنامه هاى
يك مطالعه اوليه با هدف تعيين ميزان آكاهى زنان از علائم هشدار

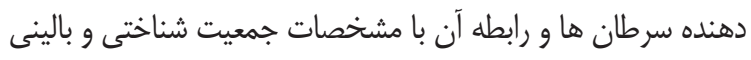

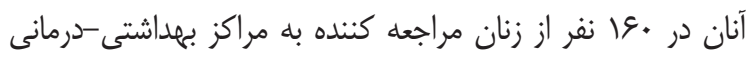
شهرى تحت يوشش دانشخاه علوم يزشكى بوشهر انجام شد. نتايج

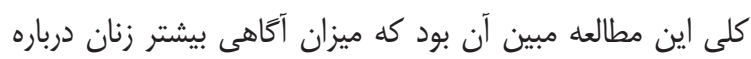
علايم هشدار دهنده سرطان ها در حد ضعيف بوده است. به علان بلاوه، سطح تحصيلات زنان تنها متغير مهم تاثير كَار بر ميزان آَّاهى آنان

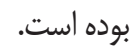
اكر خه ميانكَين سطح آكَاهى زنان از علائم هشداردهنده سرطان ها



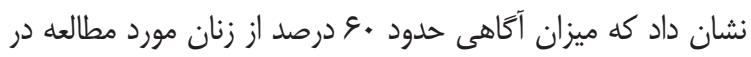
حد ضعيف بوده است. همحنين بيشتر از نيمى از زنان اظهار كردند كه



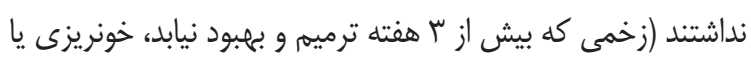

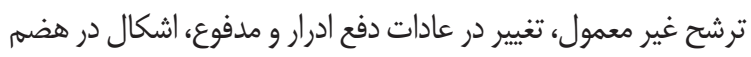
و جذب غذا، سرفه هاى مكرر و يا خشونت و تغيير در صدا). شايد

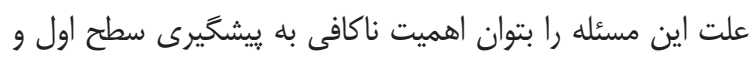


مردم دربارة سرطان و آموزش درباره عوامل خطر، اهميت بالايى دارد. در يك مطالعه توصيفى-مقطعى مبتنى بر جمعيت درباره ريسك

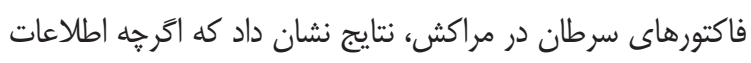


خودَزارشى آَّاهى و فراموشى يا عدم دقت كافى ياسخ دهندكًان

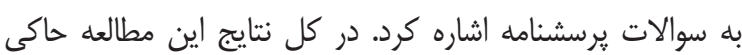
از آن است كه زنان مورد مطالعه از علايم هشدار دهنده سرطان آكاهى كافى نداشتند. اين امر ضرورت مداخلات مناسب يرستارى را براى افزايش آَاهى آن ها و آموزش با شيوه هاى نوين را از

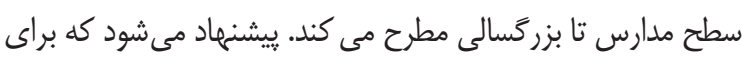

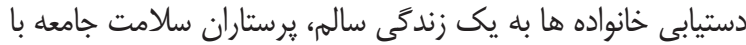

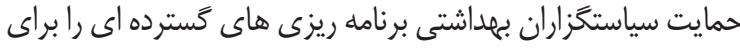
طراحى مداخلات آموزشى مناسب در سطح جامعه انجام دهند.

\section{نتيجه كيرى}

با توجه به سطح آكاهى پاييين زنان درباره علايم هشدار دهنده


جامعه و اجراى مداخلات آموزشى مؤثر در زمينه علايم هشداردهنده سرطان با كاربرد روش هاى فعال تدريس تحت حمايت سياستخزاران بهداشتى بيش از كذشته احساس مى شود. بنابراين يافته هاى اين

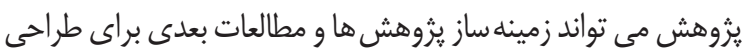
مداخلات آموزشى و اجراى آن ها در سطح جامعه باشد.

\section{تشكر و فدر دانى}

اين يزوهش حاصل طرح تحقيقاتى مصوب كميته سازمانى اخلاق

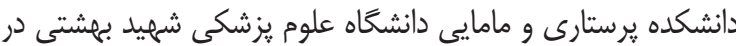
مى IR.SBMU.PHNM.1299.339 تهران با كدانس باشد. بدين وسيله از استاد محترم سركار خانم دكتر كامليا روحانى و مسئولان مراكز بهداشتى- درمانى شهرستان بوشهر و زنان مراجعه كننده به مراكز بهداشتى - درمانى كه ما را در انجام اين يزوهش

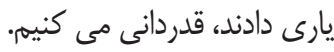

\section{References}

1. Hirokawa, K., Utsuyama, M., Kikuchi, Y. and Kitagawa, M. Scoring of immunological vigor: trial assessment of immunological status as a whole for elderly people and cancer patients. In: Pawelec G, editor. Immunosenescence. New York: Springer; 2007, pp.15-23.

2. Jemal, A., Bray, F., Center, M.M., Ferlay, J., Ward, E. and Forman, D. Global Cancer Statistics. Cancer American: A Cancer Journal for Clinicians. 2011. 61(2), pp.69-90.

3. Sadjadi, A. Mehdi, N., Ali, M., Alireza, M.J.,
غربالخَّى در زنان ساروى در حد متوسط و در زنان ساكن فسا ضعيف

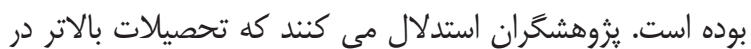
واحدهاى يثوهش علت اين اختلاف بوده است (حدود وثا درصد از زنان سارى و זr درصد از زنان ساكن در شهر فسا، داراى تحصيلات

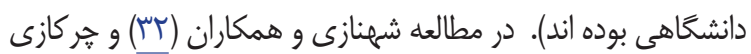
و همكاران (سّ) نيز نتايج مشابجىى وجود داشت، و افزايش ميزان أكاهى افراد با سطح تحصيلات بالاتر در آنان به طور معنى دارى ارتباط داشت. نتايج تحقيقات در كشورهاى توسعه يافته نشان مى إنى

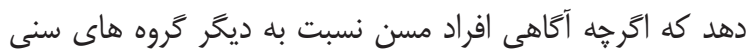
كمتر بوده، اما ارتباطى يكنواخت با روندى افزايشى بين سن افراد إداد و شناخت آن ها از سرطان ها و علائم هشدار دهنده وجود داشته

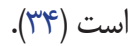
در مطالعه حاضر ساير متغيرهاى جمعيت شناختى -بالينى در بيشيبنى تغييرات ميزان آكاهى اين گروهاز زنان اثركذار نبودند. شايد بتوان به بهان دلايل متفاوتى از جمله تفاوت هاى متدولوزيك بين مطالعات و اثر

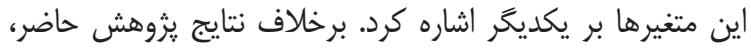


تأهل يك متغير مؤثر بر آكاهى افراد از علائم هشداردهنده سرطان

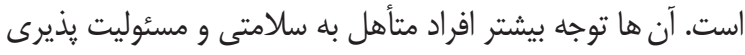
آنان را در قبال خانواده از جمله دلايل بالاتر بودن سطح آكاهى افراد ادراد متأهل در مطالعه خود معرفى كردند. به علاوه در مطالعات بيشين

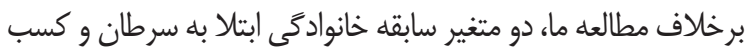
اطلاعات قبلى درباره سرطان ها نيز در افزايش ميزان آكاهى افراد

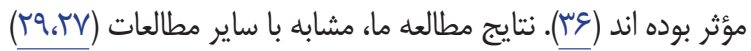
نشان داد كه دو متغير رفتارى، يعنى استعمال دخانيات و نوشيدن الكل بر ميزان آكاهى زنان از علائم هشداردهنده سرطان ها تأثير



از محدوديت هاى اين مطالعه مى توان به استفاده از برسشنامه

Reza, M. and Parkin, D.M. Cancer occurrence in Iran in 2002, an international perspective. Asian Pacific Journal of Cancer Prevention. 2005. 6(3), p.359.

4. Askarian M, Mansour Ghanaie R, Karimi A, Habibzadeh F. Infectious diseases in Iran: a bird's eye view. Clinical Microbiology and Infection. 2012 Nov 1;18(11):1081-8.

5. Ramezani, b, d. Jafarzadeh, e. Samadi, p. Malek Zadeh, R. Sajjadi, a. S. cancer incidence and mortality in community-based cancer registry in Iran from 2004 to 2006 the first report. 2009 
[Persian]

6. Notification of the Ministry of Health and Medical Education media- base, 2012 available $<$ Http://behdasht.gov.ir/?siteid=1\&pageid $>$ [Accessed December 16, 2012].

7. Young, R.C. and Wilson, C.M. Cancer Prevention Past, Present, and Future. Clinical Cancer Research, 2002. 8(1), pp.11-16.

8. Robb, K., Stubbings, S., Ramirez, A., Macleod, U., Austoker, J., Waller, J., Hiom, S. and Wardle, J. Public awareness of cancer in Britain: a population-based survey of adults. British Journal of Cancer, 2009. 101, pp.S18-S23.

9. Alberto .L. Del Valle, M.O., López, S., FayaOrnia, G. and López, M.L. Study protocol of a randomized controlled trial to improve cancer prevention behaviors in adolescents and adults using a web-based intervention supplemented with SMS. BioMed Center Public Health, 2013. 13(1), p.357.

10. Thomas, A, Kruzel N.D. Cancer: Awareness, Prevention \& Natural Therapies. Rockwood natural medicine clinic, Scottsdale, Arizona, 2009. Available: $<$ http://www .Rockwood natural medicine. com /cancer 2009.htm> [Accessed April 7, 2014].

11. Jamali, C. et al., 2010. awareness of the warning signs of cancer and its risk factors in the population over 18 years old Tehran University of Medical Sciences, 2010. Volume 17 (4), p. 296-307.

12. San Turgay, A., Sarı, D. and Türkistanlı, E.Ç. Knowledge, attitudes, risk factors, and early detection of cancer relevant to the schoolteachers in Izmir, Turkey. Preventive Medicine, 2005.40(6), pp.636-641.

13. Mazloomy Mahmoodabad SS, Noorbala MT, Rahaee Z, Mohammadi M. Knowledge, attitude and performance study of secondary school teachers of Yazd city regarding skin cancer. Journal of the European Academy of Dermatology and Venereology. 2010. 1; 24(4):424-8. [Persian]

14. McMenamin, M., Barry, H., Lennon, A.M., Purcell, H., Baum, M., Keegan, D., McDermott, E., O’Donoghue, D., Daly, L. and Mulcahy, H. A survey of breast cancer awareness and knowledge in a Western population: lots of light but little illumination. European Journal of Cancer, 2005.
41(3), pp.393-397.

15. Zareban, y. Heydar Nia, A.R. And Rakhshani, F.. The effect of educational program on HIV and AIDS awareness and performance Chabahar sailors. University of Medical Sciences, 2005.8 (1), pp. 29- 32. [Persian]

16. Sharifirad, G. Hazave, M.M. Hassanzadeh, A. et al. Effect of health education based on health belief model on preventive measures of smoking in school, 2008.10 (1), S.79- 86. [Persian]

17. MacKian, S.C. What the papers say: Reading therapeutic landscapes of women's health and empowerment in Uganda. Health \& place, 2008. 14(1), pp.106-115.

18. Mirzazadeh, S. Women and AIDS in Iran: from denial to acceptance, Health Women Magazine, 135, summer 2006. [Persian]

19. Neghab, M., Habibi, M., Rajaeefard, A. and Choobineh, A. Home Accidents in Shiraz during a 3-year Period (2000-2002). Journal of Kermanshah University of Medical Sciences, 2008. 11(4). [Persian]

20. Parvizi, S., Seyed Fatemi, M. and Kiani, K. Family dynamism \& women's health. Women Social and Psychologic Studies Quarterly, 2009.7(2), pp.54-7. [Persian]

21. Bakhtar Aghdam, F. et al. The effect of health belief model-based education on improving breast cancer screening in women's beliefs and behaviors referred to health centers of Tabriz. Medical Journal of Tabriz University of Medical Sciences, 2011.13 (6), S.25-31. [Persian]

22. Plichta, S.B., Kelvin, E.A. and Munro, B.H. Munro's statistical methods for health care research. Wolters Kluwer Health/Lippincott Williams \& Wilkins. Philadelphia, 2013.

23. Miles, A., Waller, J., Hiom, S. and Swanston, D. SunSmart? Skin cancer knowledge and preventive behaviour in a British population representative sample. Health Education Research, 2005. 20(5), pp.579-585.

24. Hajizadeh, A. and Asghar, D. Looking at the way research methods and statistical analysis in the life sciences and health. Publications Tehran, Tehran, 2011. [Persian]

25. Nunnally J.C. \& Bernstein I.H. Psychometric Theory. New York: McGraw-Hill, 1994.

26. Lewis, S.L., Dirksen, S.R., Heitkemper, M.M. 
and Bucher, L. Medical-Surgical Nursing: Assessment and Management of Clinical Problems, 9th edition. Elsevier Health Sciences, Philadelphia, 2014.

27. Gammage, K.L., Francoeur, C., Mack, D.E. and Klentrou, P. Osteoporosis health beliefs and knowledge in college students: the role of dietary restraint. Eating behaviors, 2009.10(1), pp.65-67.

28. Montazeri, A., Vahdaninia, M., Harirchi, I., Harirchi, A.M., Sajadian, A., Khaleghi, F., Ebrahimi, M., Haghighat, S. and Jarvandi, S. Breast cancer in Iran: need for greater women awareness of warning signs and effective screening methods. Asia Pacific Family Medicine, 2008. 7(1), p.6.

29. Lykins, E.L., Graue, L.O., Brechting, E.H., Roach, A.R., Gochett, C.G. and Andrykowski, M.A. Beliefs about cancer causation and prevention as a function of personal and family history of cancer: a national, population $\square$ based study. Psycho $\square$ Oncology, 2008. 17(10), pp.967974.

30. Godazandeh, a. Khani, H. Khalilian, A.R. Attard, G. Firuzi, M.A. et al., Women over 15 years of knowledge and practice about breast cancer preventionMedical University of Surrey. 2006. [Persian]

31. Rastad, h. Shokohi., L. Dehghani, S.l. And Moetamed Jahromy, M., The knowledge and practice of women than breast cancer in 2012, Fasa University of Medical Sciences Journal, 2012.3 (1), p. 75-80.

32. Shahnaz, e. Sabooteh, SA. Sharifirad, c. Mir Karimi, K. And Hassanzadeh, a. The effect of education on health belief model of anxiety in nulliparous pregnant women. Journal of Education and Health Promotion,.2015. 4. [Persian]

33. Charkazi, A., Samimi, A., Razzaghi, K., Kouchaki, G.M., Moodi, M., Meirkarimi, K., Kouchaki, A.M. and Shahnazi, H. Adherence to recommended breast cancer screening in Iranian Turkmen women: the role of knowledge and beliefs. International Scholarly Research Notices Preventive Medicine, 2013. [Persian]

34. Abreu, M.A.M.M., Pimentel, D.R., Silva, O.M., Blachman, I.T., Michalany, N.S., Hirata, C.H.,
Weckx, L.L. and Alchorne, M.M. Carcinoma espinocelular do lábio: avaliação de fatores prognósticos. Brazilian Dental Journal, 2004. 70 (6), pp.765-70.

35. Jamali, C. et al., 2010. awareness of the warning signs of cancer and its risk factors in the population over 18 years old Tehran University of Medical Sciences, Birjand University of Medical Sciences 2010. 17 (4), p. 296-307. [Persian]

36. Lynch, L. and Happell, B. Implementation of clinical supervision in action: part 2: implementation and beyond. International Journal of Mental Health Nursing, 2008.17(1), pp.65-72. 